\title{
Quality Appraisal of Systematic Reviews on the Efficacy and Safety of Labour Induction Methods: Systematic Review.
}

\author{
Ryan Chow ${ }^{1}$, Allen $\mathrm{Li}^{2}$, Nicole $\mathrm{Wu}^{1}$, Morgan Martin ${ }^{1}$, Jocelyn Wessels ${ }^{1}$, and Warren \\ Foster $^{1}$ \\ ${ }^{1}$ McMaster University Faculty of Health Sciences \\ ${ }^{2}$ Affiliation not available
}

May 28, 2020

\begin{abstract}
Background: The induction of labour has been increasing over the last decade. It is most often indicated when the safety of the baby or mother may be compromised. Objectives: This study aims to assess the quality of systematic reviews that examined the efficacy and/or safety of various methods of induction of labour. Search Strategy: An electronic database search of MEDLINE, Embase, and the Cochrane Library was conducted. The search strategy can be found in the online supplement. Selection Criteria: Systematic reviews that examined various methods of induction of labour. Inclusion and exclusion criteria can be found in the main text. Data Collection and Analysis: Study characteristics such as journal and impact factor, year of publication, source of funding, citation rate, etc. were retrieved. Quality assessment was conducted using A Measurement Tool to Assess Systematic Reviews (AMSTAR). Main Results: There were no significant relationships between mean AMSTAR score and number of citations $(\mathrm{p}=0.0875, \mathrm{r}=0.25 ; 95 \% \mathrm{CI},-0.04$ to 0.50$)$, journal impact factor $(\mathrm{p}=0.2959, \mathrm{r}=-0.15 ; 95 \% \mathrm{CI}$, -0.42 to 0.14 ), or publication year $(\mathrm{p}=0.5827, \mathrm{r}=0.08 ; 95 \% \mathrm{CI},-0.20$ to 0.36$)$. Cochrane studies on average scored higher than non-Cochrane studies $(\mathrm{p}=0.01)$. No significant differences were detected between the AMSTAR scores of government and nongovernment funded studies $(\mathrm{p}=0.34)$. Conclusions: Better adherence to the Preferred Reporting Items for Systematic Reviews and Meta-Analyses statement and for peer reviewers to appraise new systematic reviews with methodological assessment tools would enhance confidence in review conclusions.
\end{abstract}

\section{Introduction}

Induction of labour has been increasing over the last decade accounting for a significant proportion of deliveries $^{1}$. The induction of labour is most often indicated for a variety of reasons such as post-term pregnancy (defined as [?]42 weeks gestation), prelabour rupture of the amniotic membranes, or when the safety of the baby or mother may be compromised by a prolonged pregnancy ${ }^{2}$. Methods for induction of labour include the use of prostaglandins, oxytocin, mifepristone, nitric oxides, membrane sweeping, catheters, amniotomies, and other non-traditional methods. The method of induction depends on hospital protocol, national guidelines, and clinical factors ${ }^{3}$. Each method has advantages and drawbacks with implications for the well-being of the mother during and after parturition. Since decisions regarding induction can be time-sensitive, it is critical to define the efficacy and safety of the methods employed.

Evidence-based medicine is defined as using the best available evidence to inform clinical decision-making ${ }^{4}$. Systematic reviews and meta-analyses sit at the top of the hierarchy of evidence and integrate the best available evidence to aid in clinical-decision making ${ }^{5}$. However, when multiple systematic reviews are available and synthesis of their conclusions is not undertaken with methodological rigour, the reliability of conclusions and thus the validity of decisions arising from them may suffer. In the absence of a thorough assessment of study quality and combining the knowledge gleaned from multiple systematic reviews it becomes increasingly 
difficult for clinicians, policy-makers, and other knowledge users to draw reliable conclusions. To guide a minimal standard for preparing systematic reviews and meta-analyses, the Preferred Reporting Items for Systematic Reviews and Meta-analyses (PRISMA) statement was developed and published in 2009. PRISMA is a 27-item checklist of reporting items that are deemed essential for transparent conduct and reporting in systematic reviews ${ }^{6}$. In addition to PRISMA, the Assessing the Methodological Quality of Systematic Reviews (AMSTAR) tool was developed to evaluate the methodological quality of a systematic review and meta-analysis ${ }^{7}$. AMSTAR is an 11-item validated and reliable tool that has been used in numerous studies to evaluate the quality of systematic reviews. It is important to adhere to both checklists to ensure transparent reporting. This systematic review fulfills the PRISMA criteria, as well as the AMSTAR criteria where applicable.

\section{Objectives}

Given the prominence of labour induction in clinical practice, the quality of systematic reviews to guide induction needs to be conducted using robust and valid methods. Therefore, the goal of our study was to evaluate the quality of evidence surrounding labour induction using the AMSTAR tool.

The primary aim of this report was to investigate the quality of systematic reviews on the efficacy and safety of different methods for the induction of labour in peer-reviewed journals. The secondary aim was to examine whether different publication characteristics (e.g. intervention type, impact factor of the journal, number of citations, funding source, year of publication) were associated with the quality of the systematic review.

\section{Methods}

This systematic review was conducted according to PRISMA guidelines ${ }^{6}$ (Appendix S1 Pg. 6-7).

\section{Search Strategy}

A comprehensive electronic database search of MEDLINE, Embase, and the Cochrane Library of Systematic Reviews from inception until March $5^{\text {th }}, 2020$ was conducted. A medical librarian aided in synthesizing and validating a search strategy. Search terms include, labour or labor, induce or induction, and systematic review. The complete search strategy can be found in the online supplement (Appendix S2 Pg. 8).

\section{Eligibility Criteria}

Search results were uploaded into the Covidence software for systematic reviews (Veritas Health Innovation Ltd). Articles with duplicate titles were removed and a two-stage independent screening process was used to select studies for inclusion. Pilots were run for the initial stage of screening until review authors (N.W. and M.M.) reached a kappa agreement value of 0.8. Subesequently, reviewers independently screened titles and abstracts. Articles deemed eligible at this stage moved onto full-text screening. Discrepancies that arose during both stages of screening were resolved by joint-discussion among the authorship team until concensus was achieved. The inclusion criteria involved: (1) self-identified as a systematic review in the title or abstract; (2) systematic review must examine primary literature; (3) systematic review must investigate the safety, efficacy, and/or effectiveness of any method of induction of labour. The exclusion criteria involved: (1) nonintervention systematic reviews (e.g. diagnostic or screening tools); (2) non-english literature; (3) non-human based studies; (4) systematic reviews of systematic reviews; (5) conference abstracts and outdated reviews (when an updated version was avaialble and included); (6) reviews intended to examine the current state of literature, where participant outcomes were not the outcome of interest; and (7) conference abstracts and meta-analyses that did not incorporate a systematic review.

\section{Data Collection and Analysis}

Data for each included study including publication and methodological details: journal name and impact factor (from Clarivate Analytics), year of publication, country of the corresponding author, source of funding (e.g. government, philanthropic, industry, etc.), conflict of interest statement (dichotomous), citation rate (number of Google Scholar citations) and type of intervention studied (e.g. prostaglandins, oxytocin, 
misoprostol, etc.) were independently extracted by two authors (N.W. and M.M.) (Table S1 Pg 1-3). Discrepancies were resolved by discussion and consensus among the authorship team. The list of excluded studies can be found in the online supplement (Table S2 Pg. 4).

\section{Assessment of Methodological Quality}

Two review authors (N.W and M.M.) independently assessed the quality of studies using the AMSTAR quality assessment tool. Scores were tracked using Microsoft Excel (Redmond, Wash.). Review authors selected either "yes," "no," "not applicable," or "can't answer" for each of the 11 AMSTAR criteria. Differences in the assessment were resolved by consensus with the primary author. One point was given to a study for each of the 11 AMSTAR criteria that received a "yes," while no points were awarded for "no," "not applicable," or "can't answer". Thus, the highest total score possible was 11 (Table S3 Pg. 4-6).

\section{Strategy for Data Synthesis}

Graphs and tables created with Microsoft Excel (Redmond, Wash.) were used to summarize data. Statistical analyses were conducted with GraphPad Prism (version 7.0, GraphPad Software, Inc, USA). Pairwise correlations (AMSTAR Score vs. Impact Factor, AMSTAR Score vs. Total \# of Citations, AMSTAR Score vs. Publication Year) were evaluated using the Pearson correlation coefficient ( $\mathrm{r}$ ). The difference in AMSTAR Score by source of funding (government vs. institution, Cochrane vs. non-Cochrane, etc.) was evaluated with a two-tailed T-test. A P-value of less than 0.05 was considered statistically significant.

\section{Results}

\section{Search Results}

The electronic searches identified 387 publications, 10 (2.6\%) of which were duplicates (Figure 1). 377 articles proceeded to title/abstract screening with $323(84 \%)$ being deemed ineligible. 54 (14\%) full-text articles were retrieved and subjected to another round of screening from which $4(1.0 \%)$ studies were excluded as they were not systematic reviews of interventions and $2(0.52 \%)$ studies did not investigate the induction of labour. Finally, 48 studies $(13 \%)$ met the a prioriinclusion/exclusion criteria and were included $^{8-55}$.

\section{General Study Characteristics}

Study characteristics are summarized in Table S1. The majority of articles $(\mathrm{n}=18(38 \%))$ were published by authors from the United Kingdom, with the second most $(\mathrm{n}=8(17 \%))$ originating from the United States. The majority of the articles $(\mathrm{n}=36(75 \%))$ were published in the Cochrane Library with the second most $(\mathrm{n}=4$ $(8.3 \%)$ ) being published in the American Journal of Obstetrics and Gynecology. Government funding was involved in the largest proportion of the publications $(\mathrm{n}=16(33 \%))$, and philanthropic funding was reported for the fewest number of articles $(\mathrm{n}=2(4.1 \%))$. Some articles did not report any source of funding $(\mathrm{n}=12$ $(25 \%)$ ). The use of prostaglandins for the induction of labour was the method most frequently evaluated $(\mathrm{n}=9(19 \%))$, whereas many methods of induction were only reported in a single systematic review.

\section{Overall Methodological Quality of the Included Studies}

The distribution of AMSTAR scores for includeed studies pubished between the years $2000-2020$ had a mean (SD) of $6.75 \pm 1.6$ (Figure 2A). Adherence to each specific AMSTAR criteria was inconistent across included studies (Figure 2B). The top three most reported AMSTAR criteria included the assessment of the quality of included studies in the systematic review (Criterion 7: $\mathrm{n}=45(94 \%)$ ), the inclusion of the characteristics of the included studies (Criterion 6: $\mathrm{n}=44(92 \%)$ ), and a tie between an a priori design being provided and appropriate methods used to pool studies in a meta-analysis (Criterion $1 \&$ 9: $\mathrm{n}=40$ (83\%)). The AMSTAR criteria that were least frequently reported involved the inclusion of grey literature (Item $4: \mathrm{n}=3(6 \%)$ ), the assessment of publication bias (Item 10: $\mathrm{n}=6(13 \%)$ ), and the performance of a comprehensive literature search (Item 3: $\mathrm{n}=18(38 \%))$. The overall methodological quality was variable with scores from $3 / 11(\mathrm{n}=2)$ to $9 / 11(\mathrm{n}=7)$.

\section{Factors Associated with Methodological Quality}


No significant relationships between mean AMSTAR score and number of citations (Figure 3A; $\mathrm{p}=0.0875$, $\mathrm{r}=0.25 ; 95 \% \mathrm{CI},-0.04$ to 0.50 ), journal impact factor (Figure 3B; $\mathrm{p}=0.2959, \mathrm{r}=-0.15 ; 95 \%$ CI, -0.42 to 0.14 ), or publication year (Figure 3C; $\mathrm{p}=0.5827, \mathrm{r}=0.08 ; 95 \% \mathrm{CI},-0.20$ to 0.36 ) were found. Cochrane studies on average scored higher than non-Cochrane studies $(\mathrm{p}=0.01)$ (Figure 3D). No significant differences $(\mathrm{p}=0.34)$ were found between the AMSTAR scores of Government and philanthropic, or institution-funded studies (Figure 3E).

\section{Discussion}

\section{Main Findings}

Our findings revealed a moderate level of methodological quality for systematic reviews on the induction of labour, with no significant changes in quality over the past two decades. We further found poor adherence to AMSTAR criteria involving the inclusion of grey literature, the lack of assessment of publication bias, and failure to document the performance of a comprehensive literature search. Furthermore, the AMSTAR score for individual systematic review assessed in our study was not significantly related to the total number of citations to date, impact factor of the journal in which the article appeared, or the year of publication.

\section{Interpretation}

Systematic reviews are considered the highest level of evidence regarding the efficacy, effectiveness and safety of prophylactic or therapeutic interventions and play a significant role in guiding health policy, best practices, and evidence-based patient care. However, our quality assessment of the articles reviewed herein found that many did not utilize rigorous methodological approachs when conducting the studies. Thus the introduction of different biases that can confound conclusions and hinder policymaking, ultimately affecting patient-care cannot be excluded.

A moderate level of heterogeneity in the quality assessment of published systematic reviews involving the efficacy, effectiveness and safety of methods for the induction of labour was identified in our assessment with AMSTAR scores ranging from 3 to 9. The number of systematic reviews on this topic has increased over the past decade, with the most $(\mathrm{n}=11)$ being published in 2019. However, no significant improvement in methodological quality was found. This observation diverges from other areas in medicine, like critical care and radiology, in which an improvement in methodological quality of systematic reviews has been demonstrated over time ${ }^{56,57}$. Thus, results of our AMSTAR assessment of the published systematic reviews revealed a need for greater scientific rigour in the study of the induction of labour.

Systematic reviews funded by hospitals, institutions, philanthropists, government grants or with unreported funding had no difference in methodological quality according to the AMSTAR Checklist ( $\mathrm{p}=0.34$ ). Our findings report that only $36(75 \%)$ of the 48 studies evaluated in our assessment included a conflict of interest statement in both the systematic review and the studies included therein and thus criterion 11 of the AMSTAR Checklist was not satisfied. We suggest this particular weakness in the literature is concerning as there is a need for transparent reporting of conflicts of interest to allow for the judgement of any external influences on study conclusions. The effect of this potential bias is increased in studies that do not have an established a priori protocol where redefined outcome measures and post-hoc analysis could further exacerbate a biased perspective of the evidence. Our findings indicated that 40 of 48 articles (83\%) used an a priori study design (AMSTAR criterion \#1).

No significant correlation was observed between AMSTAR score and the journal impact factor, suggesting that leading journals may not necessarily evaluate methodological quality more rigorously than others. However, articles from the Cochrane Database of Systematic Reviews on average, score higher on the AMSTAR Checklist than articles from other peer-reviewed journals $(\mathrm{p}=0.01)$. This finding supports the generally accepted position that the methods followed by the Cochrane Collaboration sets the highest standard for conducting and publishing systematic reviews. Consistent findings regarding the quality of Cochrane reviews has also been reported for other medical disciplines as well ${ }^{58}$.

In the present assessment, there was no significant correlation between AMSTAR score and total citations. 
We sugges that this finding may indicate that authors who utilize systematic reviews to support their findings or hypotheses do not critically evaluate the quality of the studies cited. This is of particular importance if these systematic reviews are being used to generate or refute medical hypotheses.

The highest AMSTAR score of 9/11 was recorded for several Cochrane Collaboration publications $(n=7)$. Three examined the use of oxytocin ${ }^{9,11,14}$, three others examined mechanical methods of labour induction ${ }^{17,21,47}$, and one examined the use of mifepristone ${ }^{26}$. An AMSTAR point for a comprehensive literature search could not be provided for some of these reviews ${ }^{9,11,17,21,26}$. However, some ${ }^{17,26,47}$ included grey literature such as evidence from the Cochrane Pregnancy and Childbirth Group's Trials Register and clinicaltrials.gov which captures data that has not been peer reviewed. All seven high ranking articles also conducted quality assessments of the included studies, which satisfy AMSTAR criteria that we believe to be of greater importance. Important conclusions from these seven studies that scored the highest on the AMSTAR criteria include that a balloon catheter may be less effective than oral misoprostol but have a greater safety profile ${ }^{17}$. The use of a balloon catheter is likely as effective as the use of intravaginal prostaglandin $\mathrm{E}_{2}$ when inducing labour ${ }^{17}$. Oxytocin was suggested to be less effective in achieving vaginal birth within 24 hours compared to prostaglandin agents ${ }^{9}$. High-dose oxytocin $(100 \mathrm{mU}$ in the first 40 minutes, rising above $600 \mathrm{mU}$ in the first two hours) compared with low-dose oxytocin (below $100 \mathrm{mU}$ in the first 40 minutes, rising to below $600 \mathrm{mU}$ in the first two hours) has been shown to increase the risk of uterine hyperstimulation without increasing the rate of vaginal delivery within 24 hours ${ }^{14}$. However, discontinuing IV oxytocin stimulation after the active phase of labour may reduce the risk of a caesarean delivery ${ }^{11}$. Another study ${ }^{26}$ concluded that the literature is inadequate to draw conclusions that mifepristone helps induce labour. It is also suggested that the use of membrane sweeping does not provide clinical benefit and that this labour induction method should be balanced against women's discomfort ${ }^{21}$. Finally, one study concluded that there was no clear benefit from using acupuncture or acupressure in reducing the rate of caesarean section ${ }^{47}$.

\section{Strengths and Limitations}

An acknowledged strength of this study is that the PRISMA statement was adhered to and a comprehensive literature search according to the AMSTAR criteria conducted. A large scope of evidence was available and retrieved from Embase, Medline and the Cochrane Database of Systematic Reviews. An identified limitation is that the review authors who assessed the quality of the studies were aware of the journal of publication and authorship of the study. However, the potential for bias was minimized by having two independent review authors reach a consensus for each AMSTAR criteria. Another limitation involves the relatively small sample size of included studies $(\mathrm{n}=48)$. With a greater number of studies published in this area of research, new trends regarding methodological quality and publication details may emerge. Furthermore, the comparison between AMSTAR score and the number of citations may be affected by publication date of the systematic review; those recently published will not have accrued as many citations as older publications, even though they may have higher AMSTAR scores. However, we have chosen to utilize this metric as it may provide insight to how the methodological quality of the publication has affected current policymaking.

\section{Conclusions}

We conclude that, in general, methodological procedures of systematic reviews of the induction of labour literature are of moderate quality. The key criteria affecting AMSTAR scores were failure of study authors to document the performance of a comprehensive literature search, the inclusion of grey literature, as well as the absence of a statement describing the assessment of publication bias. Systematic reviews from the Cochrane Library generally had the most methodological rigour.

\section{Disclosure of Interests}

There are no conflicts of interest or financial disclosures for any authors.

\section{Contribution to Authorship}

RC planned the study, executed the search strategy, created figures, synthesized the results and drafted the manuscript. AL planned the study, conducted all statistical analysis, created figures, and edited the 
manuscript. NW reviewed articles for inclusion and exclusion criteria, conducted the quality assessment, and data extraction. MM reviewed articles for inclusion and exclusion criteria, conducted the quality assessment, and data extraction. JW and WF interpreted, analyzed the results, and edited the manuscript.

\section{Details of Ethics Approval}

Ethics approval was not obtained as this study involved a secondary analysis of previously published studies.

\section{Funding}

Salary support for JMW was provided by the CIHR Fellowship Awards.

\section{Acknoledgements}

We wish to thank Ms. Denise Smith for her assistance in preparing the search strategy.

\section{References}

1. NHS Maternity Statistics, England 2017-18 - NHS Digital [Internet]. NHS Digital. 2020 [cited 20 May 2020]. Available from: https://digital.nhs.uk/data-and-information/publications/statistical/nhs-maternitystatistics/2017-18 2. Delaney M, Roggensack A. No. 214-Guidelines for the Management of Pregnancy at $41+0$ to $42+0$ Weeks. Journal of Obstetrics and Gynaecology Canada. 2017;39(8):e164-e174. 3. Mozurkewich E, Chilimigras J, Berman D, Perni U, Romero V, King V et al. Methods of induction of labour: a systematic review. BMC Pregnancy and Childbirth. 2011;11(1). 4. Sackett D, Rosenberg W, Gray J, Haynes R, Richardson W. Evidence based medicine: what it is and what it isn't. BMJ. 1996;312(7023):71-72. 5. Montori VM, Saha S, Clarke M. A call for systematic reviews. J Gen Intern Med. 2004;19(12):1240-124115610335 6. Liberati A. The PRISMA Statement for Reporting Systematic Reviews and Meta-Analyses of Studies That Evaluate Health Care Interventions: Explanation and Elaboration. Annals of Internal Medicine. 2009;151(4):W. 7. Shea B, Grimshaw J, Wells G, Boers M, Andersson N, Hamel C et al. Development of AMSTAR: a measurement tool to assess the methodological quality of systematic reviews. BMC Medical Research Methodology. 2007;7(1). 8. Alfirevic Z, Aflaifel N, Weeks A. Oral misoprostol for induction of labour. Cochrane Database of Systematic Reviews. 2014(6). 9. Alfirevic Z, Kelly AJ, Dowswell T. Intravenous oxytocin alone for cervical ripening and induction of labour. Cochrane Database of Systematic Reviews. 2009(4). 10. Avdiyovski H, Haith-Cooper M, Scally A. Membrane sweeping at term to promote spontaneous labour and reduce the likelihood of a formal induction of labour for postmaturity: a systematic review and meta-analysis. Journal of obstetrics and gynaecology : the journal of the Institute of Obstetrics and Gynaecology. 2019;39(1):54-62. 11. Boie S, Glavind J, Velu AV, Mol BWJ, Uldbjerg N, de Graaf I, et al. Discontinuation of intravenous oxytocin in the active phase of induced labour. Cochrane Database of Systematic Reviews. 2018(8). 12. Boulvain M, Kelly AJ, Irion O. Intracervical prostaglandins for induction of labour. Cochrane Database of Systematic Reviews. 2008(1). 13. Bricker L, Luckas M. Amniotomy alone for induction of labour. Cochrane Database of Systematic Reviews. 2000(4). 14. Budden A, Chen LJY, Henry A. High-dose versus low-dose oxytocin infusion regimens for induction of labour at term. Cochrane Database of Systematic Reviews. 2014(10). 15. Carbone L, De Vivo V, Saccone G, D'Antonio F, Mercorio A, Raffone A, et al. Sexual Intercourse for Induction of Spontaneous Onset of Labor: A Systematic Review and Meta-Analysis of Randomized Controlled Trials. Journal of Sexual Medicine. 2019;16(11):1787-95. 16. de Los Reyes SX, Sheffield JS, Eke AC. Single versus Double-Balloon Transcervical Catheter for Labor Induction: A Systematic Review and Meta-Analysis. American journal of perinatology. 2019;36(8):790-7. 17. de Vaan MDT, ten Eikelder MLG, Jozwiak M, Palmer KR, Davies-Tuck M, Bloemenkamp KWM, et al. Mechanical methods for induction of labour. Cochrane Database of Systematic Reviews. 2019(10). 18. De Vivo V, Carbone L, Saccone G, Magoga G, De Vivo G, Locci M, et al. Early amniotomy after cervical ripening for induction of labor: a systematic review and metaanalysis of randomized controlled trials. American journal of obstetrics and gynecology. 2019(3ni, 0370476). 19. Dodd JM, Crowther CA. Misoprostol for induction of labour to terminate pregnancy in the second or third trimester for women with a fetal anomaly or after intrauterine fetal death. Cochrane Database of Systematic Reviews. 2010(4). 20. Dodd JM, Crowther CA, Grivell RM, Deussen AR. Elective repeat caesarean section versus induction of labour for women with a previous caesarean birth. 
Cochrane Database of Systematic Reviews. 2017(7). 21. Finucane EM, Murphy DJ, Biesty LM, Gyte GML, Cotter AM, Ryan EM, et al. Membrane sweeping for induction of labour. Cochrane Database of Systematic Reviews. 2020(2). 22. French L. Oral prostaglandin E2 for induction of labour. Cochrane Database of Systematic Reviews. 2001(2). 23. Ghosh A, Lattey KR, Kelly AJ. Nitric oxide donors for cervical ripening and induction of labour. Cochrane Database of Systematic Reviews. 2016(12). 24. Grobman WA, Caughey AB. Elective induction of labor at 39 weeks compared with expectant management: a meta-analysis of cohort studies. American journal of obstetrics and gynecology. 2019;221(4):304-10. 25. Hamidi O, Quist-Nelson J, Xodo S, Berghella V. Membrane sweeping in patients planning a trial of labor after cesarean: a systematic review and meta-analysis of randomized controlled trials. The journal of maternal-fetal \& neonatal medicine : the official journal of the European Association of Perinatal Medicine, the Federation of Asia and Oceania Perinatal Societies, the International Society of Perinatal Obstetricians. 2019(101136916):1-8. 26. Hapangama D, Neilson JP. Mifepristone for induction of labour. Cochrane Database of Systematic Reviews. 2009(3). 27. Hofmeyr GJ, Gulmezoglu AM, Pileggi C. Vaginal misoprostol for cervical ripening and induction of labour. Cochrane Database of Systematic Reviews. 2010(10). 28. Howarth G, Botha DJ. Amniotomy plus intravenous oxytocin for induction of labour. Cochrane Database of Systematic Reviews. 2001(3). 29. Hutton EK, Mozurkewich EL. Extra-amniotic prostaglandin for induction of labour. Cochrane Database of Systematic Reviews. 2001(2). 30. Kavanagh J, Kelly AJ, Thomas J. Sexual intercourse for cervical ripening and induction of labour. Cochrane Database of Systematic Reviews. 2001(2). 31. Kavanagh J, Kelly AJ, Thomas J. Breast stimulation for cervical ripening and induction of labour. Cochrane Database of Systematic Reviews. 2005(3). 32. Kavanagh J, Kelly AJ, Thomas J. Hyaluronidase for cervical ripening and induction of labour. Cochrane Database of Systematic Reviews. 2006(2). 33. Kavanagh J, Kelly AJ, Thomas J. Corticosteroids for cervical ripening and induction of labour. Cochrane Database of Systematic Reviews. 2006(2). 34. Kelly AJ, Alfirevic Z, Ghosh A. Outpatient versus inpatient induction of labour for improving birth outcomes. Cochrane Database of Systematic Reviews. 2013(11). 35. Kelly AJ, Kavanagh J, Thomas J. Relaxin for cervical ripening and induction of labour. Cochrane Database of Systematic Reviews. 2001(2). 36. Kelly AJ, Kavanagh J, Thomas J. Castor oil, bath and/or enema for cervical priming and induction of labour. Cochrane Database of Systematic Reviews. 2013(7). 37. Kenyon S, Tokumasu H, Dowswell T, Pledge D, Mori R. High-dose versus low-dose oxytocin for augmentation of delayed labour. Cochrane Database of Systematic Reviews. 2013(7). 38. Kim SW, Nasioudis D, Levine LD. Role of early amniotomy with induced labor: a systematic review of literature and meta-analysis. American Journal of Obstetrics and Gynecology MFM. 2019;1(4):100052. 39. Luckas M, Bricker L. Intravenous prostaglandin for induction of labour. Cochrane Database of Systematic Reviews. 2000(4). 40. Middleton P, Shepherd E, Flenady V, McBain RD, Crowther CA. Planned early birth versus expectant management (waiting) for prelabour rupture of membranes at term (37 weeks or more). Cochrane Database of Systematic Reviews. 2017(1). 41. Muzonzini G, Hofmeyr GJ. Buccal or sublingual misoprostol for cervical ripening and induction of labour. Cochrane Database of Systematic Reviews. 2004(4). 42. Nasioudis D, Kim SW, Schoen C, Levine LD. Maternal and neonatal outcomes with mechanical cervical dilation plus misoprostol compared to misoprostol alone for cervical ripening; a systematic review of literature and metaanalysis. American Journal of Obstetrics and Gynecology MFM. 2019;1(2):101-11. 43. Nishi D, Shirakawa MN, Ota E, Hanada N, Mori R. Hypnosis for induction of labour. Cochrane Database of Systematic Reviews. 2014(8). 44. Ornat L, Alonso-Ventura V, Bueno-Notivol J, Chedraui P, Perez-Lopez FR, Health O, et al. Misoprostol combined with cervical single or double balloon catheters versus misoprostol alone for labor induction of singleton pregnancies: a meta-analysis of randomized trials. The journal of maternal-fetal \& neonatal medicine : the official journal of the European Association of Perinatal Medicine, the Federation of Asia and Oceania Perinatal Societies, the International Society of Perinatal Obstetricians. 2019(101136916):1-16. 45. Saccone G, Della Corte L, Maruotti GM, Quist-Nelson J, Raffone A, De Vivo V, et al. Induction of labor at full-term in pregnant women with uncomplicated singleton pregnancy: A systematic review and meta-analysis of randomized trials. Acta obstetricia et gynecologica Scandinavica. 2019;98(8):958-66. 46. Smith CA. Homoeopathy for induction of labour. Cochrane Database of Systematic Reviews. 2003(4). 47. Smith CA, Armour M, Dahlen HG. Acupuncture or acupressure for induction of labour. Cochrane Database of Systematic Reviews. 2017(10). 48. Sotiriadis A, Petousis S, Thilaganathan B, Figueras F, Martins WP, 
Odibo AO, et al. Maternal and perinatal outcomes after elective induction of labor at 39 weeks in uncomplicated singleton pregnancy: a meta-analysis. Ultrasound in obstetrics \& gynecology : the official journal of the International Society of Ultrasound in Obstetrics and Gynecology. 2019;53(1):26-35. 49. Thomas J, Fairclough A, Kavanagh J, Kelly AJ. Vaginal prostaglandin (PGE2 and PGF2a) for induction of labour at term. Cochrane Database of Systematic Reviews. 2014(6). 50. Thomas J, Kelly AJ, Kavanagh J. Oestrogens alone or with amniotomy for cervical ripening or induction of labour. Cochrane Database of Systematic Reviews. 2001(4). 51. Vogel JP, Osoti AO, Kelly AJ, Livio S, Norman JE, Alfirevic Z. Pharmacological and mechanical interventions for labour induction in outpatient settings. Cochrane Database of Systematic Reviews. 2017(9). 52. Vogel JP, West HM, Dowswell T. Titrated oral misoprostol for augmenting labour to improve maternal and neonatal outcomes. Cochrane Database of Systematic Reviews. 2013(9). 53. Wei S, Wo BL, Qi HP, Xu H, Luo ZC, Roy C, et al. Early amniotomy and early oxytocin for prevention of, or therapy for, delay in first stage spontaneous labour compared with routine care. Cochrane Database of Systematic Reviews. 2013(8). 54. West HM, Jozwiak M, Dodd JM. Methods of term labour induction for women with a previous caesarean section. Cochrane Database of Systematic Reviews. 2017(6). 55. Zamawe C, King C, Jennings HM, Mandiwa C, Fottrell E. Effectiveness and safety of herbal medicines for induction of labour: A systematic review and meta-analysis. BMJ Open. 2018;8(10):e022499. 56. Tunis A, McInnes M, Hanna R, Esmail K. Association of Study Quality with Completeness of Reporting: Have Completeness of Reporting and Quality of Systematic Reviews and Meta-Analyses in Major Radiology Journals Changed Since Publication of the PRISMA Statement?. Radiology. 2013;269(2):413-426. 57. Delaney A, Bagshaw S, Ferland A, Manns B, Laupland K, Doig C. Critical Care. 2005;9(5):R575. 58. Campbell J, Kavanagh S, Kurmis R, Munn Z. Systematic Reviews in Burns Care. Journal of Burn Care \& Research. 2017;38(2):e552-e567.

\section{Hosted file}

2020_05_27_Figures.docx available at https://authorea.com/users/327268/articles/454871quality-appraisal-of-systematic-reviews-on-the-efficacy-and-safety-of-labour-inductionmethods-systematic-review 